\title{
High fidelity simulator experience for enhancing communication effectiveness: Applications to quality and safety education for nurses
}

\author{
Elaine Della Vecchia, , Lisa Sparacino \\ Department of Nursing, School of Health Professions, New York Institute of Technology, New York, United States
}

Received: April 26, 2015

DOI: $10.5430 /$ jnep.v5n9p78

\author{
Accepted: May 28, 2015 \\ Online Published: June 23, 2015 \\ URL: http://dx.doi.org/10.5430/jnep.v5n9p78
}

\begin{abstract}
The United States Joint Commission identified miscommunication as the main cause of unexpected injuries and mortality not related to the patient's medical condition. One strategy for improving information transfer and inter-professional communication is the Situation-Background-Assessment-Recommendation (SBAR) communication model. The purpose of this study was to test the effects of exposure to a real time high fidelity simulation experience. A quasi-experimental design, consisting of one treatment group was conducted. A purposeful sample of $\mathrm{N}=45$ respondents was drawn from students registered in an undergraduate level clinical course. Pre-tests and post-tests based on the American Association of Colleges of Nursing's (AACN's) Quality and Safety in Nursing Education (QSEN) were administered to measure if exposure to the simulation training impacted knowledge and attitudes regarding communication in the delivery of patient care. Results based on a paired t-test revealed an 8.27 point increase in scores after exposure to the intervention. This outcome was highly significant $(d f=44, t=-4.86, p=.000)$. Although a few students did not appear to benefit, results suggest that exposure to the SBAR model during a high fidelity simulation experience is generally a successful method for enhancing student knowledge and attitudes regarding effective communication in health care.
\end{abstract}

Key Words: Situation-Background-Assessment-Recommendation, Simulation, Communication, Medication, Patient safety, Quality and safety education for nurses

\section{INTRODUCTION}

\subsection{Introduction to the problem}

The current health care industry increasingly challenges health care professionals to provide safe patient care. Despite the advances in technology, basic communication remains an essential aspect of delivering safe and effective patient care. Ineffective communication among health care providers is a major contributing factor to adverse patient outcomes. Communication errors are the root cause of most injuries and deaths not related to the natural cause of the patient's ill- ness. ${ }^{[1]}$ According to the Joint Commission, ${ }^{[2]}$ an estimated 80 percent of serious medical errors, inclusive of medication mistakes, wrong site surgery, and delays in treatment are caused by miscommunication at several different levels. The Institute of Medicine estimates there are approximately 1.5 million medication errors per year. ${ }^{[3]}$ Administration errors account for $26 \%-32 \%$ of all medication errors. Administration errors result from a number of factors including errors related to prescribing, transcribing, dispensing, and administration of the medication. The improvement of the

\footnotetext{
*Correspondence: Elaine Della Vecchia; Email: edellave@nyit.edu; Address: Department of Nursing, School of Health Professions, New York Institute of Technology, New York, United States.
} 
effectiveness of communication among healthcare providers has been classified as a 2009 US National Patient Safety Goal. $^{[4]}$

One strategy for improving inter-professional communication is the Situation-Background-AssessmentRecommendation (SBAR) communication model. ${ }^{[5]}$ The Joint Commission and the Institute for Healthcare Improvement (IHI) both advocate the use SBAR as a tool to assist health care providers to enhance communication resulting in improved effectiveness in information transfer to patient and practitioner populations.

\subsection{Situation-background-assessment-recommendation}

SBAR is a communication format initially developed by the military and refined by the aviation industry to reduce risks associated with the transmission of inaccurate and incomplete information. SBAR provides a predictable structure for promoting critical thinking and inter-professional communication. ${ }^{[1,5]}$ Knowledge to know what to communicate, as well as how and when to communicate patient information must not be capricious. The use of SBAR strengthens the transfer process by providing a structure that allows information to be timely and structured. ${ }^{[6]}$ The IHI and the Joint Commission both support the use of SBAR as a form of structured communication between patients and practitioners. ${ }^{[4]}$ The use of a formal communication tool such as SBAR creates a culture that promotes safety in patient care and prevents undesirable results such as patient care errors. ${ }^{[7]}$ SBAR serves as a template for inter-professional communication. In recent years, the health care industry has borrowed the practice of using checklists from the aviation industry, which well ensures that nothing is missed in high-risk situations. ${ }^{[5]}$ SBAR has also been proven as an effective teaching tool. Although many students learn the SBAR technique they often lack the ability to utilize it. ${ }^{[8]}$ Incorporating the SBAR model into teaching and learning situations using role playing provides students with the opportunity to increase confidence and ability in communicating concisely, relaying pertinent information in a timely manner. ${ }^{[8]}$ When used by nurses to communicate with each other during hands off report and with other members of the health care team, the SBAR model proved to help nurses remain focused and increased the ability to exchange a high volume of information safely and accurately. ${ }^{[6]}$ It also improves critical reasoning allowing for critical information to be relayed clearly and concisely in half the time. ${ }^{[6,7]}$ SBAR has been proven to be a standard communication tool that improves clinical judgment among nursing students. The SBAR model is recommended to be used by educators as a framework for teaching, learning, and assessment, decreasing vagueness during introduction

Published by Sciedu Press of new information, and communication between student and faculty. ${ }^{[9]}$ It has been recommended that further studies be conducted regarding the use of the SBAR model to enhance students' and nurses' ability to reach a decision regarding patient care and communicate it in an effective, timely manner. ${ }^{[6,9]}$

\section{METHOD}

\subsection{Materials studied}

Dayton and Henriksen ${ }^{[10]}$ identified individual, group, and organizational factors related to communication failures. They recommend structured and explicitly designed forms of communication for health care providers with different communication styles. More efficient and accurate encoding and decoding of verbal messages reduce risk of misinterpretation associated with patient safety. Both verbal and nonverbal forms of communication are an integral part of inter-professional and nursing care situations. It is critical to ensure that information is accurately interpreted by all members of the health care team.

Standardizing the structure of critical communication with a model such as SBAR assists the speaker to organize thoughts, prepare critical information, and allows for more attention to be focused on the critical points of the message. ${ }^{[11]}$ The American Association of Colleges of Nursing (AACN) ${ }^{[12]}$ has implemented the Quality and Safety in Nursing Education (QSEN) as a priority initiative to decrease patient care errors. QSEN competencies ${ }^{[13]}$ were established to provide an opportunity for nursing faculty to develop ways in which education can occur across the cognitive, psychomotor, and affective domains. AACN has charged nurse educators with the mission to develop teaching strategies and programs which foster knowledge, skills, and attitudes regarding effective communication with an emphasis on patient safety.

Scholars have suggested that the use of high fidelity simulation is an effective means to teach patient safety and communication. In a study conducted by Marshall and Harrison, ${ }^{[14]}$ medical students demonstrated improvements in information transfer when using the SBAR method during high fidelity simulation learning experiences. Krautscheid ${ }^{[15]}$ also reported an increase in information transfer among nursing students using the SBAR model. In the past, teaching quality and safety focused more attention on skills and knowledge acquisition and had less priority on communication strategies. SBAR competencies have been used to enhance quality and safety in the health care industry and health care education. ${ }^{[15]}$ Few current evidence-based publications specifically examine the use of high fidelity simulation experiences on improving communication to prevent practice errors as outlined by the SBAR model. 
The purpose of this study was to evaluate if exposure to the SBAR Model incorporated into real time high fidelity simulation experiences had an impact on effective communication among nursing students. This project follows the recommendation of the National League for Nursing, which encourages nursing faculty to research innovative teaching and learning strategies. ${ }^{[16]}$

\subsection{Methodology}

This project used a quasi-experimental design to determine if the use of SBAR model during a high fidelity simulation experience enhances communication skills between practitioners and with patients. The hypothesis was therefore H1: Exposure to SBAR Model during high fidelity simulation significantly increases effective communication skills among nursing students. A purposeful sample of student nurses was drawn from an undergraduate clinical nursing course in a baccalaureate program. Coded numbers were assigned to each respondent to assure that responses for all aspects of data collection remained anonymous. Study participants were exposed to a high fidelity simulation intervention using SBAR training involving a patient with iatrogenic hyperkalemia.

The patient was ordered $140 \mathrm{mEqs}$ of potassium over four hours. This dose of potassium is higher than the average recommended maximum dose. The students were expected to recognize the potassium and other medication errors, and communicate the situation effectively to the health care provider. Students had to consider several factors including equipment, environment, team work, staff, institutional context, management, and the patient when deciding where the miscommunication occurred. The students then participated in a high fidelity simulation experience which concentrated on the use of the SBAR Model during medication administration. At the end of the simulation, the respondent completed a post-test considering the same factors.

The tests administered utilized the AACN's QSEN Tool developed by McMillan. ${ }^{[17]}$ The tool was pilot tested and received a Kappa value of $\alpha=.81$ for internal validity. A score ranging from a low of 0 to a high of 100 cumulative points can be achieved. Each participant completed a pre-test immediately prior to the simulation experience, and completed a post-test immediately following the simulation experience. A paired t-test was conducted to measure differences in pre and post exposure, and to determine if differences were statistically significant. The significance level was held standard at $p \leq .05$. Case summaries were also reviewed for bi-directionality to identify the proportion of scores that increased versus declined.

\section{RESULTS}

\subsection{QSEN Scores}

A paired-sample t-test was conducted to compare QSEN scores achieved immediately before and immediately after exposure to the intervention. Average scores achieved before revealed was $(\mathrm{M}=74.04, \mathrm{SD}=1.16)$ and after the intervention $(\mathrm{M}=82.31, \mathrm{SD}=1.28)$. The 8.27 point average increase achieved after the intervention was highly significant ( $d f=44$, $t=-4.86, p=.000)$. These results suggest that exposure to the SBAR model during a high fidelity simulation experience significantly enhanced knowledge and attitudes regarding communication. Therefore, the null hypothesis of no relationship is rejected and the alternative hypothesis is accepted (see Table 1).

Table 1. $T$-test results comparing QSEN scores pre and post exposure to sbar model during a high fidelity simulation exercise $(\mathrm{N}=45)$

\begin{tabular}{llllll}
\hline QSEN Score & Mean & SD & $\boldsymbol{t}$ & $\boldsymbol{d} \boldsymbol{f}$ & $\boldsymbol{p}$ \\
\hline Pre-Test & 74.04 & 1.16 & -4.86 & 44 & .000 \\
Post-Test & 82.31 & 1.28 & & & \\
\hline
\end{tabular}

Note. $p \leq .05=$ statistically significant.

\subsection{Bi-directionality}

Upon visual inspection of the pre and post-test scores, it was noted that the majority of students $(86.7 \%)$ experienced an increase in average scores. A small number of participants $(13.3 \%)$ experienced a decline in average scores. Approximately one in eight students appeared to earn a lower score after exposure to this training model. However, significant increases in mean scores for the entire sample supported enhancement of knowledge, attitudes, and effective communication associated with providing quality and safe patient care. Therefore, it is concluded that utilization of the SBAR model during a high fidelity simulation experience is highly valuable as a training tool for nursing students (see table 2).

\section{Discussion}

The project was implemented as a response to the Joint Commission safety standards regarding medication administration. The authors intended to improve nursing education by implementing a teaching strategy that might lead to better patient outcomes through increased knowledge and patient safety. Faculty are directed to continuously seek out teaching, learning, and assessment methodologies that improve the knowledge base and confidence level of student nurses. ${ }^{[16]}$ Also important is for faculty to disseminate the knowledge, skills, and attitudes that will lead to nurses providing safe and quality care within a diverse, ever changing health care system. ${ }^{[12]}$ The authors used the Joint Commission Safety 
Standards to provide an experience that follows QSEN recommendations, as well as Benner's et al. call for faculty to enhance nursing education standards.

Table 2. Case summaries: Pre-Test and Post-Test scores*

\begin{tabular}{|c|c|c|}
\hline & Pre-Test & Post-Test \\
\hline 1 & 70 & 87 \\
\hline 2 & 69 & 76 \\
\hline 3 & 73 & 83 \\
\hline 4 & 77 & 92 \\
\hline 5 & 67 & 75 \\
\hline 6 & 67 & 84 \\
\hline 7 & 67 & 87 \\
\hline 8 & 77 & 78 \\
\hline 9 & 77 & 95 \\
\hline 10 & 78 & 83 \\
\hline 11 & 70 & 89 \\
\hline 12 & 73 & 86 \\
\hline 13 & 74 & 83 \\
\hline 14 & 92 & 86 \\
\hline 15 & 74 & 87 \\
\hline 16 & 72 & 87 \\
\hline 17 & 67 & 80 \\
\hline 18 & 69 & 78 \\
\hline 19 & 77 & 82 \\
\hline 20 & 81 & 90 \\
\hline 21 & 80 & 90 \\
\hline 22 & 78 & 95 \\
\hline 23 & 77 & 69 \\
\hline 24 & 86 & 67 \\
\hline 25 & 73 & 63 \\
\hline 26 & 89 & 63 \\
\hline 27 & 98 & 86 \\
\hline 28 & 65 & 83 \\
\hline 29 & 71 & 72 \\
\hline 30 & 63 & 92 \\
\hline 31 & 83 & 87 \\
\hline 32 & 80 & 81 \\
\hline 33 & 77 & 82 \\
\hline 34 & 71 & 92 \\
\hline 35 & 77 & 83 \\
\hline 36 & 71 & 95 \\
\hline 37 & 71 & 81 \\
\hline 38 & 71 & 82 \\
\hline 39 & 73 & 82 \\
\hline 40 & 77 & 76 \\
\hline 41 & 56 & 78 \\
\hline 42 & 63 & 61 \\
\hline 43 & 73 & 78 \\
\hline 44 & 75 & 98 \\
\hline 45 & 63 & 80 \\
\hline Total & 45 & 45 \\
\hline
\end{tabular}

*Limited to first 100 case.

The high fidelity simulation experience provided students with real time experiential learning utilizing technology that enhances the ability to effectively communicate in an interprofessional environment. After the nursing students were exposed to the high fidelity simulation experience, average scores on the post-test increased by 8.27 points over the pre-test. This increase strongly suggests that utilization of the SBAR Model for teaching and practicing during a high fidelity medication administration simulation is valuable not only for reducing errors, but for improving knowledge, skills, and attitudes ${ }^{[12]}$ regarding safety and the overall quality of patient care delivery.

A concern noted by the researchers was a decline in the average post-test scores experienced by approximately one in eight students. Students participated in two high fidelity simulation experiences lasting one and one half hours each and a full day of instruction. Observed declines in scores experienced by some students therefore, might be attributable to fatigue rather than the actual failure to learn skills for enhancing communication using the SBAR Model.

Faculty members are challenged with providing education which promotes the practice of safe patient care. SBAR is an effective model for assisting providers to communicate a shared set of expectations. Barnsteiner ${ }^{[18]}$ suggests that despite the healthcare culture of safety there has been less consideration to integrate the culture of safety content into the education of healthcare professionals. Patient safety education, inclusive of skills needed to process and communicate information, is needed not only early in nursing training, but is essential in all phases of nursing education and practice. This simulated experience assisted students to appreciate the need to share patient information in a clear, complete, concise and structured format. Results reinforced that the SBAR Model is an effective tool for improving communication efficiency and accuracy for enhancing nursing education and ensuring patient safety.

\section{Conclusion}

Effective communication remains a vital facet for delivery of safe quality patient care. Mortality not related to a patient's illness is directly related to ineffective communication among health care workers. ${ }^{[1]}$ The purpose of this project was to develop a teaching methodology that would allow student nurses to develop the knowledge, skills, and attributes ${ }^{[12]}$ that would lead to the advancement of essential communication skills, thereby decreasing injuries and errors responsible for adverse patient outcomes. Medication errors caused by ineffective communication are one of the leading causes of adverse patient outcomes. ${ }^{[2]}$ This study adds a teaching methodology to the cadre of teaching, learning, and assessment techniques in nursing education that allow students to gain knowledge and confidence in safe, quality patient care.

\section{CONFLiCTS OF INTEREST Disclosure}

The authors declare that there is no conflict of interest. 


\section{REFERENCES}

[1] Leonard M, Graham S, Bonacum D. The human factor: The critical importance of effective teamwork and communication in providing safe care. Quality Safe Health Care. 2004; 13: 185-190. http://dx.doi.org/10.1136/qshc. 2004.010033

[2] The Joint Commission. New Targeted Solutions Tool Available for Hand-off Communications-Ambulatory Customers Pilot Tested. 2012.

[3] Aspden P, Wolcott JJ, Bootman L, et al. Committee on identifying and preventing medication errors: Board on health care services. Atlanta, GA: The National Academics. 2006.

[4] The Joint Commission. 2009 National patient safety goals hospital program. 2009.

[5] Haig KM, Sutton S, Whittington J. SBAR: A shared mental model for improving communication between clinicians. Journal on Quality and Patient Safety. 2006; 32(3): 167-175.

[6] Cornell P, Gervis MT, Yates L, et al. Impact of SBAR on nurse shift reports and staff rounding. MedsurgNursing. 2014; 23(5): 334-342.

[7] Chaharsoughi NT, Ahrari S, Alikhah S. Comparison the effect of teaching of SBAR technique with role play and lecturing on communication skill of nurses. Journal of Caring Sciences. 2014; 3(2): 11-147.

[8] Kesten KS. Role-play using SBAR technique to improve observed communication skill in senior nursing students. Journal of Nursing Education. 2011; 50(2): 79-87. PMid:21210611 http://dx.doi.o rg/10.3928/01484834-20101230-02

[9] Lancaster RJ, Westphal J, Jambunathan J. Using SBAR to promote clinical judgment in undergraduate nursing students, Journal of Nursing Education. 2015; 54(3 Suppl.): S31-S34. PMid:25692739 http://dx.doi.org/10.3928/01484834-20150218-08
[10] Dayton E, Henriksen K. Communication failure: Basic components, contributing factors and the call for structure. Joint Commission Journal on Quality and Patient Safety. 2007; 33(1): 34-47. PMid:17283940

[11] Wood D. SBAR improves communication in patient handoff. AMN Healthcare Inc. 2008. Available from: http://www.nursezone. com/nursing-news-events/more-features/SBAR-Improve s-Communication-in-Patient-Handoffs_21756.aspx

[12] American Association of Colleges of Nursing. Quality and Safety Education for Nurses (QSEN) Education Consortium. 2011a. Available from: http: //www . aacn. nche.edu/qsenec

[13] American Association of Colleges of Nursing. Quality and Safety Education for Nurses (QSEN). 2011b. Available from: http: //www . qsen.org/competencies.php

[14] Marshall S, Flanagan B, Harrison J. Does the teaching of a structured tool improve medical students' communication skills? The Association for the Study of Medical Education Golden Jubilee, ASM. 2007.

[15] Krautscheid LC. Improving communication among healthcare professionals: Preparing student nurses for practice. International Journal of Nursing Education Scholarship. 2008; 5: 1-13. PMid:18976237 http://dx.doi.org/10.2202/1548-923X.1647

[16] Benner P, Sutphen M, Leonard V, et al. Educating nurses: A call for radical Transformation. San Francisco, CA: Jossey-Bass; 2010.

[17] McMillan LR. Utilizing SBARR; using Peer reviewers in a low fidelity lab exercise. QSEN. 2010.

[18] Barnsteiner J. "Teaching the Culture of Safety" OJIN: The Online Journal of Issues in Nursing. 2011; 16(3): M5. 